\title{
Performance of the Double Multilayer Monochromator On the NSLS Wiggler Beam Line X25
}

\author{
Lonny E. Berman and Zhijian Yin \\ Brookhaven National Laboratory, National Synchrotron Light Source, Upton, New York 11973 \\ Steven B. Dierker and Eric Dufresne \\ University of Michigan, Department of Physics, Ann Arbor, Michigan 48109 \\ Simon G.J. Mochrie and Ophelia K.C. Tsui \\ Massachusetts Institute of Technology, Department of Physics, Cambridge, Massachusetts 02139
}

Stephen K. Burley, Fong Shu and Xiaoling Xie ${ }^{1}$

Rockefeller University, Laboratories of Molecular Biophysics, Howard Hughes Medical Institute, New York, New York 10021

Malcolm S. Capel and Robert M. Sweet

Brookhaven National Laboratory, Biology Department, Upton, New York 11973

\begin{abstract}
.
A tunable, double multilayer $x$-ray monochromator has recently been implemented on the National Synchrotron Light Source (NSLS) X25 wiggler beam line. It is based on a parallel pair of tungsten-boron-carbide multilayer films grown on silicon substrates and purchased from Osmic, Inc. of Troy, Michigan, USA. It acts as an optional alternative to the conventional double silicon crystal monochromator, and uses the same alignment mechanism. Two other NSLS beam lines also have had this kind of monochromator installed recently, following the lead of the NSLS X20C IBM/MIT beam line which has used a double multilayer monochromator for several years. Owing to the 100 times broader bandwidth of a multilayer $x$-ray monochromator, compared with a silicon monochromator, the multilayer monochromator has the obvious advantage of delivering 100 times the flux of a silicon monochromator, and thereby makes more efficient use of the continuous synchrotron radiation spectrum, yet preserves the narrow collimation of the incident synchrotron beam. In particular, multilayer x-ray bandwidths, on the order of $1 \%$, are well-matched to $x$-ray undulator linewidths. Performance results for the X25 multilayer monochromator are presented, comparing it with the silicon monochromator. Of note is its short- and long-term performance as an x-ray monochromator delivering the brightness of the wiggler source in the presence of the high-power white beam. Detailed measurements of its spatial beam profile and wavelength dispersion have been made, and it is shown how its resolution could be improved when desired. Finally, its peculiar, anisotropic resolution function in reciprocal space, and its bearing upon x-ray crystallography and scattering experiments, will be discussed, and highlighted by the results of a protein crystallography experiment.
\end{abstract}

\section{INTRODUCTION}

Most experimental applications of synchrotron radiation require monochromatic beams, which are usually produced by perfect silicon or germanium crystal monochromators. Such monochromators diffract a photon energy bandwidth of typically $10^{-4}$, producing concomitant relative energy or wavevector resolutions. Often, such fine resolution is unnecessary for monochromatic beam applications such as small-angle scattering, absorption, and emission experiments. Broader bandwidth diffractive optics, such as layered synthetic microstructures (henceforth referred to as multilayers), consisting of a periodic film of alternating heavy and light layers grown atop a substrate for which

1) Current address: Vertex Pharmaceuticals, Inc., Cambridge, Ma.

CP417, Synchrotron Radiation Instrumentation: Tenth US National Conference edited by Ernest Fontes

(C) 1997 The American Institute of Physics 1-56396-742-1/97/\$10.00 
the resultant bilayers form "Bragg planes", can be designed to diffract a photon energy bandwidth of $10^{-2}$ or greater $[1,2]$. This results in a "monochromatic" beam that is at least 100 times as intense as a beam monochromated by a perfect silicon or germanium crystal, making more efficient use of the continuous synchrotron radiation spectrum. In particular, multilayer bandwidths are very well matched to the radiated linewidths of modern x-ray undulators, and hence multilayers offer the prospect of perfect line filtering for such insertion devices [3]. While mosaic crystal monochromators can be conceived to diffract broad bandwidths $[4,5]$, they do not preserve the synchrotron source brightness. Since multilayers in principle function as perfect, specularly-reflecting diffractors, they do preserve the brightness.

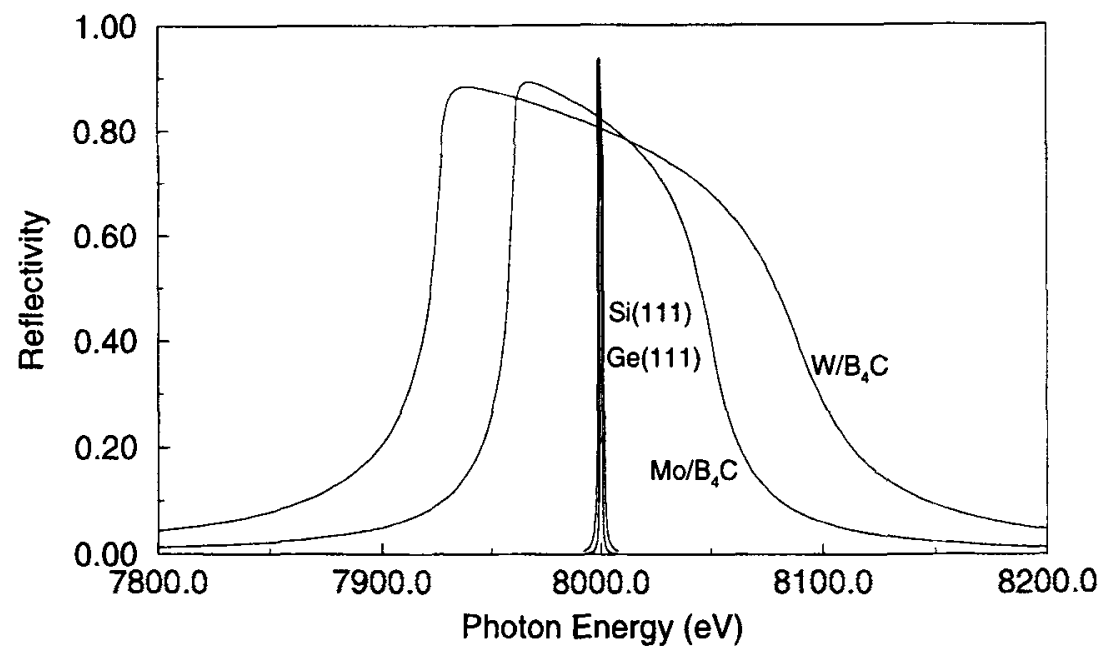

FIGURE 1. The theoretical x-ray reflectivities, at $8 \mathrm{keV}$, of semi-infinite perfect crystal Bragg peaks of $\mathrm{Si}(111)$ and $\mathrm{Ge}(111)$, and first-order multilayer Bragg peaks of $\mathrm{Mo} / \mathrm{B}_{4} \mathrm{C}$ and $\mathrm{W} / \mathrm{B}_{4} \mathrm{C}$ (with fabrication parameters described in the text), are compared.

A comparison of the diffractive properties of perfect multilayers vs. perfect single crystals is shown in Fig. 1. First-order reflectivity curves are presented, for a nominal photon energy of $8 \mathrm{keV}$, for a $\mathrm{Si}(111)$ crystal, a $\mathrm{Ge}(111)$ crystal, a semi-infinite $\mathrm{Mo} / \mathrm{B}_{4} \mathrm{C}$ multilayer of $\mathrm{d}$-spacing $25 \AA$ (for which the thickness of the heavier Mo component of each bilayer is $30 \%$ of the $25 \AA$ bilayer thickness), and a semi-infinite $\mathrm{W} / \mathrm{B}_{4} \mathrm{C}$ multilayer with identical d-spacing and thickness attributes to the $\mathrm{Mo} / \mathrm{B}_{4} \mathrm{C}$ multilayer [6]. Whereas the relative bandwidth of the $\mathrm{Si}(111)$ curve is $1.3 \times 10^{-4}$ and that of the $\mathrm{Ge}(111)$ curve is $3.3 \times 10^{-4}$, the relative width of the $\mathrm{Mo} / \mathrm{B}_{4} \mathrm{C}$ curve is $1.2 \times 10^{-2}$ and that of the $\mathrm{W} / \mathrm{B}_{4} \mathrm{C}$ curve is $2.0 \times 10^{-2}$, about 100 times the widths of the $\mathrm{Si}(111)$ and $\mathrm{Ge}(111)$ curves. The bandwidth $\mathrm{B}$ of a perfect Bragg diffractive optic is given by

$$
B=C d^{2} \frac{|F|}{v}
$$

where $C$ is a proportionality constant, $d$ is the $\mathrm{d}$-spacing of the Bragg planes, $F$ is the structure factor for the Bragg reflection, and $v$ is the unit cell volume. The ratio of $|F|$ to $v$ is simply an effective $\mathrm{x}$-ray scattering density for the material and Bragg reflection at hand. It is easy to see that the Ge(111) bandwidth is larger than the Si(111) width by virtue of its higher scattering density (the d-spacings of these crystal Bragg planes are about the same), and similarly for the $\mathrm{W} / \mathrm{B}_{4} \mathrm{C}$ width in comparison with the $\mathrm{Mo} / \mathrm{B}_{4} \mathrm{C}$ width. The bandwidths of the multilayers are broader than those for the crystals by virtue of their larger $\mathrm{d}$-spacings. The reflectivity of the perfect semi-infinite multilayers can be calculated using the same Darwin-Prins formula that is applicable to perfect semi-infinite crystals, with a suitable definition of a structure factor for the multilayer's Bragg planes which takes into account the bilayer thickness and composition profile [1]. Reference 7 presents a comprehensive review of the state-of-the-art of the fabrication and use of $\mathrm{x}$-ray multilayers.

\section{X25 DOUBLE MULTILAYER MONOCHROMATOR}

A double multilayer monochromator, consisting of a pair of W/Si multilayers, was implemented on the IBM/MIT $\mathrm{X} 20 \mathrm{C}$ bending magnet beam line at NSLS over 10 years ago, primarily for time-resolved x-ray scattering experiments 
for which high monochromatic beam intensity was desired but high monochromaticity was unnecessary [8]. This beam line has been highly sought and oversubscribed by users since.

Single multilayer elements were tested for their diffractive properties under very high power, and power density, loads on the NSLS X25 wiggler beam line [9], using its focussed white beam, a few years later [10]. Tests showed that commonly-used multilayers such as $\mathrm{W} / \mathrm{Si}$ and $\mathrm{W} / \mathrm{B}_{4} \mathrm{C}$, grown on $\mathrm{Si}$ substrates, could well survive incident power densities of as much as $150 \mathrm{~W} / \mathrm{mm}^{2}$ and have their diffractive properties preserved over the course of beam exposure, provided that they could be suitably cooled and contained in a proper inert gas or vacuum environment in order to prevent chemical attack of the multilayer coating.

Subsequently, x-ray scattering experiments were carried out on X25 using a single W/Si multilayer element (borrowed from the $\mathrm{X} 20 \mathrm{C}$ beam line) as a monochromator, placed in the experimental hutch just upstream of the sample and cooled in the same manner (at room temperature) described in Ref. 10. Success with this simple arrangement was attained in high angle scattering experiments [11], but not in small angle scattering experiments [12], owing to the substantial low angle diffuse scattering from the single multilayer element (which was impacted by white x-ray beam) and close distance of the sample and detector to it. It rapidly became clear that an experimental arrangement similar to that employed on beam line X20C, i.e. placing the multilayer monochromator in the shielded beam line enclosure far upstream of the experimental hutch, and using a double multilayer monochromator setup instead of a single one (to suppress diffuse scattering) which would additionally afford convenient energy tunability without needing to tilt or elevate the experimental diffractometer downstream, would be highly desirable on X25.

Several $\mathrm{W} / \mathrm{B}_{4} \mathrm{C}$ multilayer elements were purchased from Osmic, Inc. of Troy, Michigan, USA [13] for use on X25 initially and other NSLS beam lines later. Each element consists of 120 bilayers, each of thickness (d-spacing) about $25 \AA$ with the heavy $\mathrm{W}$ component's thickness being about $30 \%$ of the d-spacing. The multilayer films were grown on optically-flat $\mathrm{Si}$ crystal wafers of roughness $<1 \AA$ and slope error $<1$ arcsec, and with dimensions $50 \mathrm{~mm} \times 25 \mathrm{~mm} \times 5 \mathrm{~mm}$. The elements were designed to fit readily into the standard X25 double crystal monochromator tank using the same exact fixtures and cooling block that silicon crystals are normally mounted on, thereby facilitating interchange. At a typical photon energy of use of $9 \mathrm{keV}$, the multilayer Bragg angle is $1.6 \mathrm{deg}$. The standard offset height of the X25 monochromatic beam from the white beam is $25 \mathrm{~mm}$. To preserve this height with a pair of diffractive elements set to a Bragg angle of $1.6 \mathrm{deg}$ requires that they be separated, along the beam path, by $45 \mathrm{~cm}$. The largest separation permitted within the monochromator tank is less than half of this, $20 \mathrm{~cm}$. Accomodation of the multilayer pair within the tank required us to reduce the offset height of the doubly-diffracted monochromatic beam to be nominally $6 \mathrm{~mm}$ which, for the $1.6 \mathrm{deg}$ Bragg angle, cut down the separation along the beam path to a manageable $11 \mathrm{~cm}$ value, and at the same time permitted a reasonable range of tunability. The $50 \mathrm{~mm}$ multilayer element length is just large enough to intercept the full incident beam height that is reflected from the upstream focussing mirror, at the $1.6 \mathrm{deg}$ Bragg angle. The X25 monochromator design [9] allows for scanning of the perpendicular spacing between the two diffractive elements. This feature is particularly useful when multilayers are employed, for the spacing can be scanned, as the photon energy is tuned, in order to maximize the beam footprint intercept and thus the photon throughput.

With this upgrade of the monochromator tank to accomodate multilayers, small angle scattering $\mathrm{x}$-ray photon correlation spectroscopy [12], and other experimental applications requiring a multilayer monochromator, became feasible on X25.

\section{BEAM PROFILE AND WAVELENGTH DISPERSION}

Upon the first installation of a pair of $\mathrm{W} / \mathrm{B}_{4} \mathrm{C}$ multilayer elements in the X25 monochromator tank, measurements of the doubly-diffracted flux, beam profile, and wavelength dispersion functions were made, with the beam line set up in its focussed mode of operation (upstream toroidal mirror placed in the white beam path, focussing nominally at 1:1 magnification). With the NSLS x-ray ring operating at $2.584 \mathrm{GeV}, 210 \mathrm{~mA}$ current, and with the X25 27 -pole wiggler operating at a gap of $24 \mathrm{~mm}$ (giving a nominal field of $1.1 \mathrm{~T}$ ), a focussed "monochromatic" beam flux of $1.4 \times 10^{14} \mathrm{ph} / \mathrm{sec}$ was measured at $8 \mathrm{keV}$. An ionization chamber was used to determine the flux, and was carefully calibrated with filters to ensure linearity of response. In fact, it was not possible to measure the unattenuated flux with the chamber because of non-linearity of response in that flux range, so the final flux determination was made via extrapolation of the attenuated fluxes. Under these operation conditions of the NSLS x-ray ring and $\mathrm{X} 25$, the standard $\mathrm{Si}(111)$ double crystal monochromator typically delivers $2.5 \times 10^{12} \mathrm{ph} / \mathrm{sec}$ at $8 \mathrm{keV}$. Thus the flux derived with use of the multilayer monochromator represented a gain of 56 compared with that from the $\mathrm{Si}(111)$ monochromator. It was noticed that most, but not all, of the incident beam was intercepted by the multilayers; with a more careful alignment, the potential flux gain could have been even higher. The observed flux gain compared with the $\mathrm{Si}(111)$ double crystal monochromator is roughly consistent with expectation, based upon comparison of the diffracted bandwidths and reflectivities (Fig. 1), and by taking account of the fact that two successive reflections 
(o) ring current $=250 \mathrm{~mA}$, vertica height $\mathrm{FWHM}=1.6 \mathrm{~mm}$

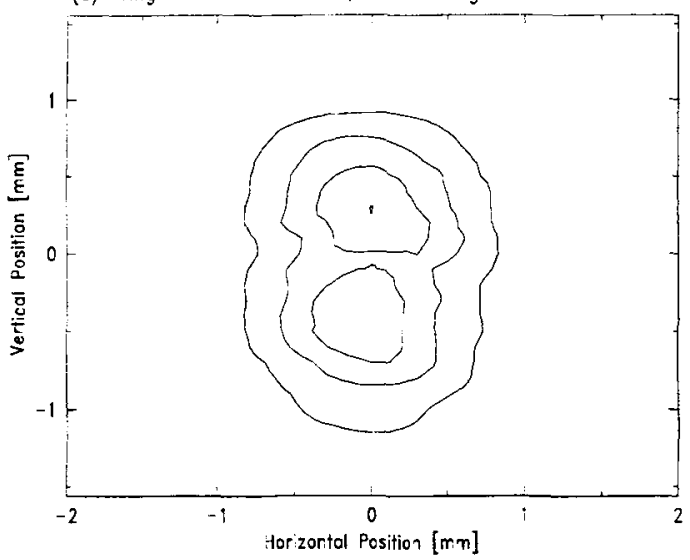

(b) ring current $=175 \mathrm{MA}$, verticoi height $\mathrm{FWHM}=1.1 \mathrm{~mm}$

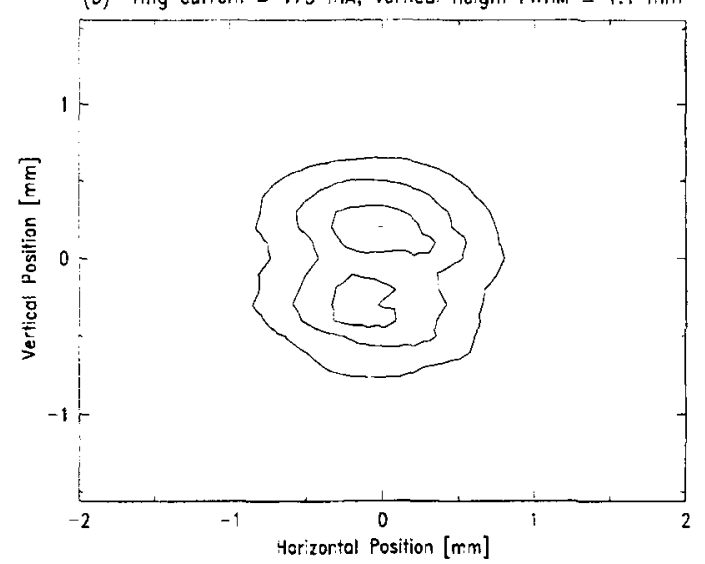

FIGURE 2. Spatial double multilayer diffracted beam profiles, at the nominal beam focus position in the X25 experimental hutch, are shown at $8 \mathrm{keV}$ with ring currents of (a) $250 \mathrm{~mA}$ and (b) $175 \mathrm{~mA}$. The contours are at $25 \%$ increments.

An important aspect of performance of the multilayer monochromator relates to its withstanding of the incident heat load. At a machine current of $300 \mathrm{~mA}$, the x-ray beam power reflected from the upstream focussing mirror and incident upon the monochromator is about $120 \mathrm{~W}$, with a density (normal to the beam direction) of $5 \mathrm{~W} / \mathrm{mm}^{2}$. It is straightforward to show that, for a freely-supported wafer, an integrated thermal bowing slope error of about $25 \mathrm{arcsec}$ in the silicon substrate of the multilayer would be induced to span the beam footprint, with the silicon nominally at room temperature, and irrespective of the substrate's thickness [14,15]. A silicon monochromator has an intrinsic rocking curve angular width that is somewhat lower than this, even for low-index Bragg reflections, and this implies that the thermal slope error is rather severe in this case. Fortunately, the intrinsic rocking curve angular width of the $\mathrm{W} / \mathrm{B}_{4} \mathrm{C}$ multilayer predicted by dynamical diffraction theory is much broader, about 125 arcsec at 8 $\mathrm{keV}$, and one might therefore expect that the thermal slope error is not problematic. This however is not truly the case, as revealed in Fig. 2, which shows spatial profiles of the multilayer-monochromated beam at the focal position in the experimental hutch at different machine currents, obtained by raster-scanning, across the beam, a pinhole placed before an ionization chamber detector. The bowing slope error in the first multilayer element spreads out the ray paths of the diffracted beam, smearing the vertical beam profile, and this effect decreases as the machine current (and thence the power load) decreases [16]. The extent of the vertical profile smearing shown in Fig. $2(1.6 \mathrm{~mm}$ FWHM at $250 \mathrm{~mA}$ current) is approximately consistent with the calculated thermal bowing, given the $10 \mathrm{~m}$ distance from the monochromator to the focal position. Extrapolation of the vertical profile widths to zero current confirms that they are principally due to thermal bowing and not to intrinsic slope error.

Profiling the beam spatially is a suitable means of determining the thermal slope error of the first multilayer in this case, because deconvolution from a measurement of the multilayer's rocking curve would be complicated by the fact that the intrinsic rocking curve width is relatively broad to begin with. Advantage is taken of the long distance from the multilayer to the beam focus, in gaining sensitivity with the profiling technique. It could be used to provide feedback to an adaptive correction system that might be designed to compensate the thermal slope error [16]. Such a system has not been implemented at X25 for multilayers, but has for silicon crystals [17], and has been shown to produce a vertical beam focus size of as small as $0.3 \mathrm{~mm}$ FWHM when the collection solid angle of the upstream focussing mirror is filled [16]. Consequently, compared with silicon crystals, the gain in flux afforded via the use of multilayers at X25 is offset somewhat by the broader spot profile which is left uncompensated.

The reflectivity of all $\mathrm{W} / \mathrm{B}_{4} \mathrm{C}$ multilayer elements purchased from Osmic had been measured by Osmic prior to delivery, using a $\mathrm{Cu} \mathrm{K}_{\alpha} \mathrm{x}$-ray source. Each of the multilayers in the most recent pair we obtained has an absolute peak reflectivity of 0.65 and a diffracted bandwidth (for the first-order Bragg peak) of $1.5 \times 10^{-2}$. The diffracted bandwidth of the multilayer pair was measured at X25 using a Si(111) analyzer crystal in the experimental hutch, and the observed dispersion function (measured at $9 \mathrm{keV}$ ) is shown in Fig. 3. Its main peak has a relative $(\Delta \mathrm{E} / \mathrm{E}$, where $\mathrm{E}$ is the nominal photon energy) FWHM of $1.1 \times 10^{-2}$, which is consistent with the product of two identical functions each with a width of $1.5 \times 10^{-2}$. The interference function side fringes about the main peak arise from the finite number of bilayers (120 for each element in this case). The calculated reflectivity curve for this double multilayer monochromator, based upon its fabrication parameters and assuming ideal interfaces, is shown in Fig. 3 , and agrees poorly with experimental measurement in terms of both peak reflectivity and width. The calculation 


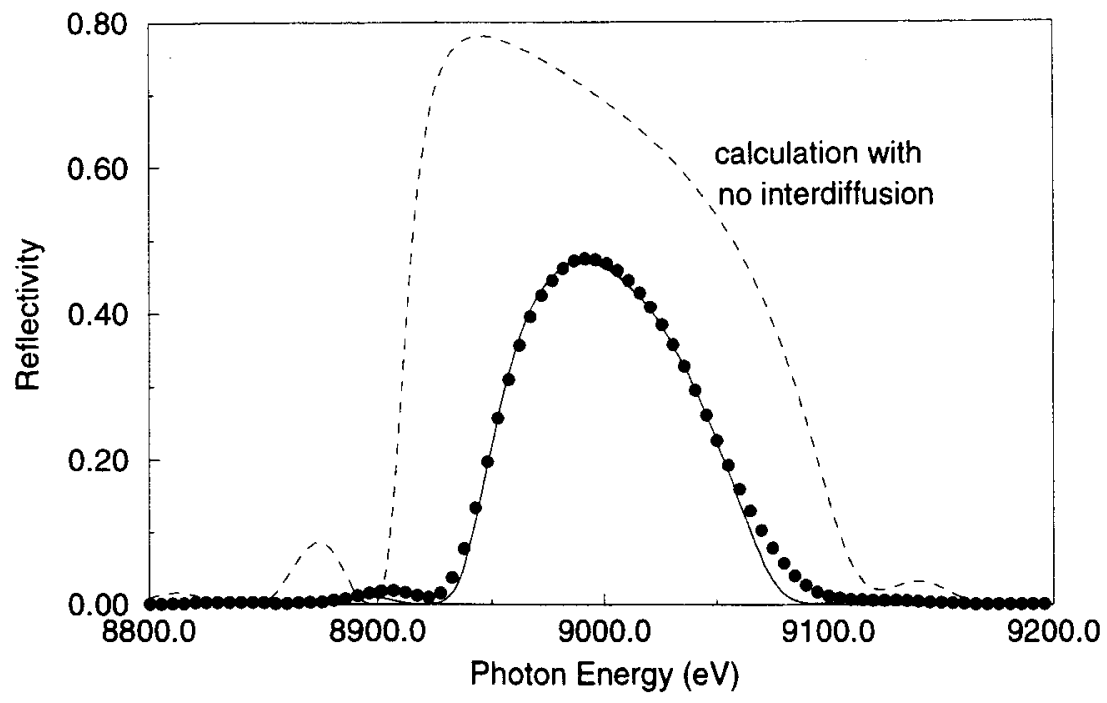

FIGURE 3. The measured double multilayer dispersion function at $9 \mathrm{keV}$ (dotted curve) is compared with theory, for the ideal case of no interdiffusion at the interfaces (dashed curve) and for the case of an interdiffusion thickness with a $\sigma$ of $4 \AA$ (solid curve).

was repeated by assuming that an interdiffusion thickness, between layers, was present with a $\sigma$ value of $4 \AA$, and this gave much better agreement with experiment as also shown in Fig. 3. This level of interdiffusion is consistent with the expectation of the vendor for this kind of multilayer. As it bears upon the reflectivity, it can be understood as a Debye-Waller effect, due to static disorder, that modulates the calculated structure factor for the case of ideal interfaces. Under dynamical diffraction, this leads mainly to a narrowing of the width of the reflectivity curve, and secondarily in some instances to a lowering of the peak reflectivity.

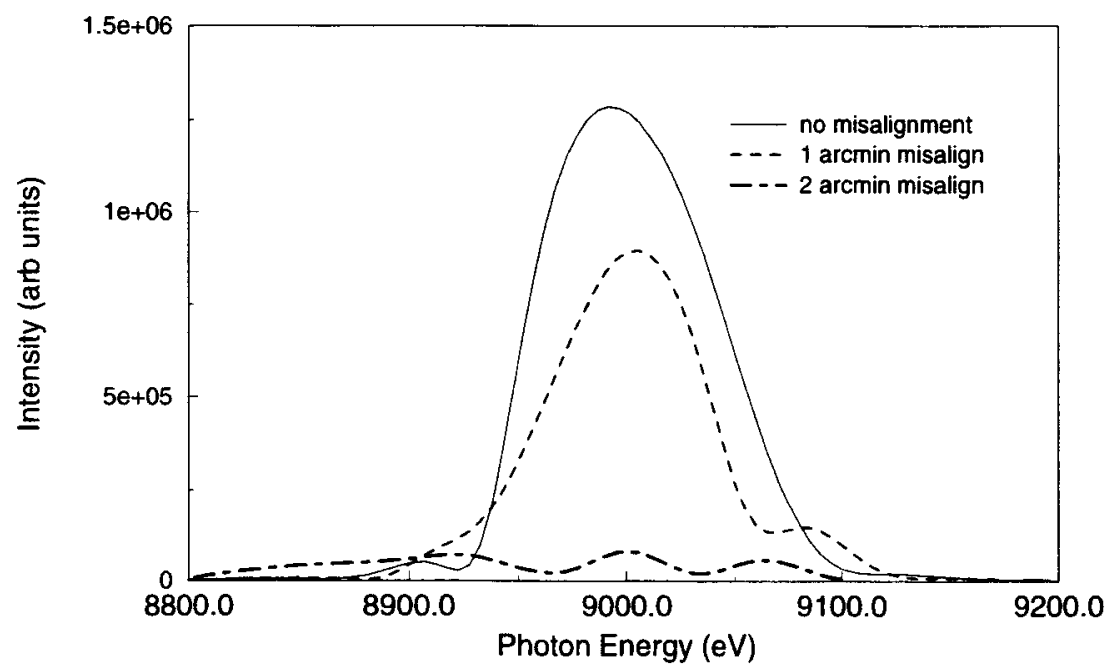

FIGURE 4. The measured double multilayer dispersion functions at $9 \mathrm{keV}$ are shown for the cases of no misalignment of the multilayers (solid curve), a misalignment of 1 arcmin (dashed curve), and of 2 arcmin (chain-dashed curve).

An important issue concerns whether a relative resolution function width narrower than $1.1 \times 10^{-2} \mathrm{FWHM}$, produced by the present pair of multilayers, is attainable, without compromise of peak reflectivity. From Eqn. 1, it seems that two pathways can be pursued to obtain a narrower bandwidth multilayer element. One involves reducing the ratio of structure factor to unit cell volume. This could be attained through use of a lower density "heavy layer", e.g. via the substitution of Mo for $\mathrm{W}$ (and which is available commercially from Osmic) or via a reduction of the thickness fraction of the "heavy layer". The other alternative is to reduce the d-spacing of the bilayer, whose effect on the bandwidth is rather sensitive because of the proportionality to $d^{2}$. In practice, the performance of these multilayers, mainly in terms of their peak reflectivity, might be compromised upon pursuit of most of these bandwidth 
reduction methods, due to a greater relative impact of the interdiffusion and roughness at the layer interfaces which are rather difficult to improve beyond present capabilities (a few $\AA$ ) $[7,13]$.

An alternative method of effective bandwidth narrowing takes advantage of the fact that multiple reflections are involved in most monochromator systems, including X25's. Just as resolution narrowing has been demonstrated with multiple silicon crystal reflections, whereby the individual silicon reflections can be slightly misaligned from parallel leaving a narrower effective transmission in terms of relative angle or wavelength spread (as well as reduced intensity) $[18,19]$, so can the principle be applied to multiple multilayer reflections. This is illustrated in Fig. 4, which shows the measured dispersion function at X25, at $9 \mathrm{keV}$, using different misalignment angles between the two multilayer elements. Marginal improvement is demonstrated, from a FWHM of $1.1 \times 10^{-2}$ to $0.9 \times 10^{-2}$, upon introduction of a misalignment angle of 1 arcmin. A misalignment angle of 2 arcmin leaves a much narrower main peak but comparable neighboring interference function side fringes, for in this case the main peak of one multilayer element overlaps the first side fringe of the other, and vice versa. The multiple fringes which result, of similar intensities, compromise the objective of bandwidth narrowing via misalignment of the multilayers. It is envisioned that this problem could be minimized via fabrication of multilayers with more bilayers to suppress the interference function fringes.

With intermittent use of the $\mathrm{W} / \mathrm{B}_{4} \mathrm{C}$ multilayer elements in the $\mathrm{X} 25$ monochromator tank over the past few years, their reflectivity properties have not been noted to degrade with continued exposure to the X25 X-ray beam. Only slight discoloration has been observed on the multilayer films at the beam footprints, mainly on the first multilayer element (which sees the white beam). The high vacuum of the X25 monochromator tank, which typically operates at a pressure of as low as $10^{-8}$ Torr, is largely responsible for minimizing the contamination of the films.

\section{APPLICATION TO X-RAY CRYSTALLOGRAPHY AND SCATTERING}

The usefulness of a multilayer monochromator in an x-ray diffraction experiment requires consideration of the resulting resolution function in reciprocal space. For simplicity, let us consider incident and diffracted (from a sample) beams to have $\delta$ function angular profiles within and perpendicular to the scattering plane; at synchrotron sources, the angular widths of the incident and scattered beams (with the latter usually defined by slits or an analyzer crystal) result in relative wavevector resolution width contributions of $10^{-4}$ to $10^{-3}$. The wavelength spread of a monochromator can be represented in reciprocal space by a pair of Ewald spheres with a common incident beam direction, and tangent at the origin of reciprocal space. The spheres have different radii corresponding to the low and high energy limits (long and short wavelength limits) of the wavelength dispersion function. For any given scattered beam direction, the finite wavelength spread gives rise to a spread of the reciprocal space resolution function in the longitudinal direction (the direction of momentum transfer), according to

$$
\frac{\Delta q}{q}=\frac{\Delta \lambda}{\lambda}
$$

where $q$ denotes the momentum transfer magnitude and $\lambda$ denotes the wavelength. It is clearly evident that, as $q$ increases, so does $\Delta \boldsymbol{q}$; at higher momentum transfers, the longitudinal reciprocal space resolution becomes poorer in absolute terms. This is shown schematically in Fig. 5 .

Since the relative wavelength resolution from a multilayer monochromator is about $10^{-2}$, this contribution to the resolution function in reciprocal space, at synchrotron sources, is generally much larger than the contributions arising from the angular spreads of the incident and scattered beams, by a factor of 10 to 100 . The resolution function can be thought of as a longitudinal streak, rather than a sharp point as is normally the case with use of a silicon or germanium monochromator. This has important implications on the application of a multilayer monochromator in x-ray diffraction experiments, with data interpretation requiring careful consideration of the resolution function. Several diffraction experiments executed at NSLS beam lines X20C and X25 have needed to consider carefully the details of this highly anisotropic resolution function in order for the data to be properly understood; a case example, a small angle scattering "speckle" experiment carried out at X25, is described in Ref. 20.

We will now focus attention on the application of a multilayer monochromator for rotation-diffraction experiments of the sort that often are performed in macromolecular crystallography. These involve rotating a single crystal in a monochromatic beam and recording the Bragg diffraction spots on a film, imaging plate, or charge-coupled device (CCD) area detector. As in any crystallography experiment, the success of a rotation-diffraction measurement depends on the capability of separating and indexing Bragg reflections, and reducing their integrated intensities. The poorer the resolution in reciprocal space, the more challenging these tasks are. The situation is shown in Fig. 5, which overlays the reciprocal lattice points corresponding to a single crystal with the Ewald spheres corresponding to the low and high energy limits of the multilayer monochromator resolution function. A rotation measurement entails rotation of the crystal's reciprocal lattice, about the origin, and a Bragg peak will appear on the detector 


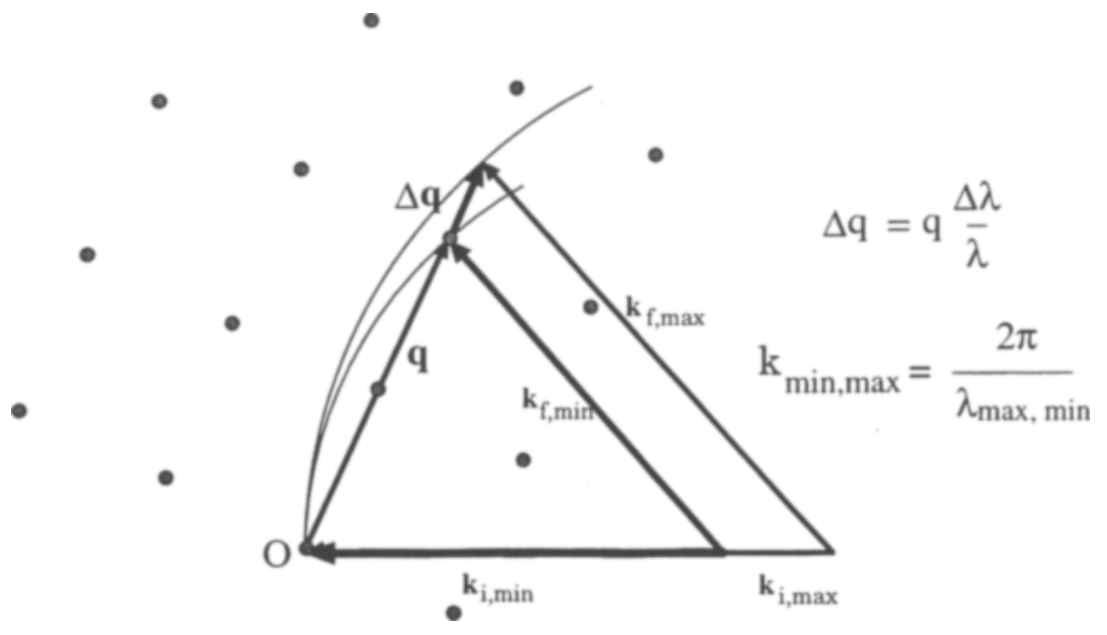

FIGURE 5. Reciprocal space representation of the scattering kinematics and resolution with use of a multilayer monochromator. $\mathbf{k}_{i, \min }$ and $\mathbf{k}_{\boldsymbol{f} \text {,min }}$ are the incident and scattered wavevectors, respectively, corresponding to the long wavelength limit of the monochromator resolution, and $\mathbf{k}_{i, \max }$ and $\mathbf{k}_{f, \max }$ are the incident and scattered wavevectors, respectively, corresponding to the short wavelength limit. Superimposed is the reciprocal lattice corresponding to a single crystal whose diffraction pattern is to be measured.

plane in a scattered beam direction that is determined by the intersection of a lattice point with any of the allowed Ewald spheres that fall within the resolution function (with the two spheres shown in Fig. 5 being the limiting spheres). As the crystal (and thereby its reciprocal lattice) is rotated, a lattice point will first cross one limiting sphere, giving rise to a diffracted beam aimed from the center of the sphere toward that point where it contacts the sphere, and ultimately the lattice point will cross the other limiting sphere, creating a diffracted beam aimed in a different direction from the center of the second sphere toward the point of contact with the sphere. The locus of points on the detector plane which traces satisfaction of the Bragg condition for all intermediate Ewald spheres (intermediate photon energies within the resolution function width) will be a radial streak with increasing length at higher $g$ values.

Separation of streaks along the radial direction on the detector plane requires that the separation between the limiting Ewald spheres in the longitudinal direction in reciprocal space must be smaller than the distance between adjacent reciprocal lattice points in that direction. This distance is just $2 \pi / a$, where $a$ is the unit cell dimension in that direction. Thus we require that

$$
\Delta q<\frac{2 \pi}{a}
$$

Given that the momentum transfer $q$ at the Bragg condition is $2 \pi / d$ with $d$ being the crystal $\mathrm{d}$-spacing corresponding to the appropriate reciprocal lattice point, we find that

$$
\frac{\Delta q}{q}<\frac{d}{a}
$$

This essentially says that the limit in relative $\mathrm{d}$-spacing resolution, $d / a$, is determined by the relative momentum transfer resolution $\Delta q / q$ or (from Eqn. 2) the relative wavelength spread $\Delta \lambda / \lambda$.

This was put to the test in a protein crystallography experiment on X25, with use of the original double multilayer pair which gave a relative wavelength resolution of $1.5 \times 10^{-2} \mathrm{FWHM}$. A crystallized heterodimer of two eukaryotic transcription factors, TAF42/TAF62, whose structure had already been solved on an NSLS bending magnet beam line [21], was studied at X25 during single-bunch operation of the NSLS x-ray ring, for which the X25 intensity is close to that available on an NSLS bending magnet beam line with a similar optical configuration under normal multi-bunch operation of the $\mathrm{x}$-ray ring. The longest unit cell dimension of this crystal is $111 \AA$, and it therefore should have been feasible to observe resolvable Bragg peaks to a $d$-spacing resolution as small as about $2 \AA$. Figure 6 shows a diffraction pattern for which the crystal rotation range was 1deg with an exposure time of 5 sec, recorded with a MAR Research $300 \mathrm{~mm}$ diameter imaging plate camera using a wavelength of $1.4 \AA$ and a detector distance of $175 \mathrm{~mm}$. Under these conditions, the nominal d-spacing resolution at the perimeter of the plate was about $1.7 \AA$. Achievement of comparable Bragg spot intensities using the bending magnet beam line, equipped with a silicon 
crystal monochromator, required exposure times of more than 1 min (during normal multi-bunch ring operation). This by itself proves that dramatic increases in intensity can be afforded with the use of a broad bandwidth multilayer monochromator compared with a narrow bandwidth silicon monochromator.

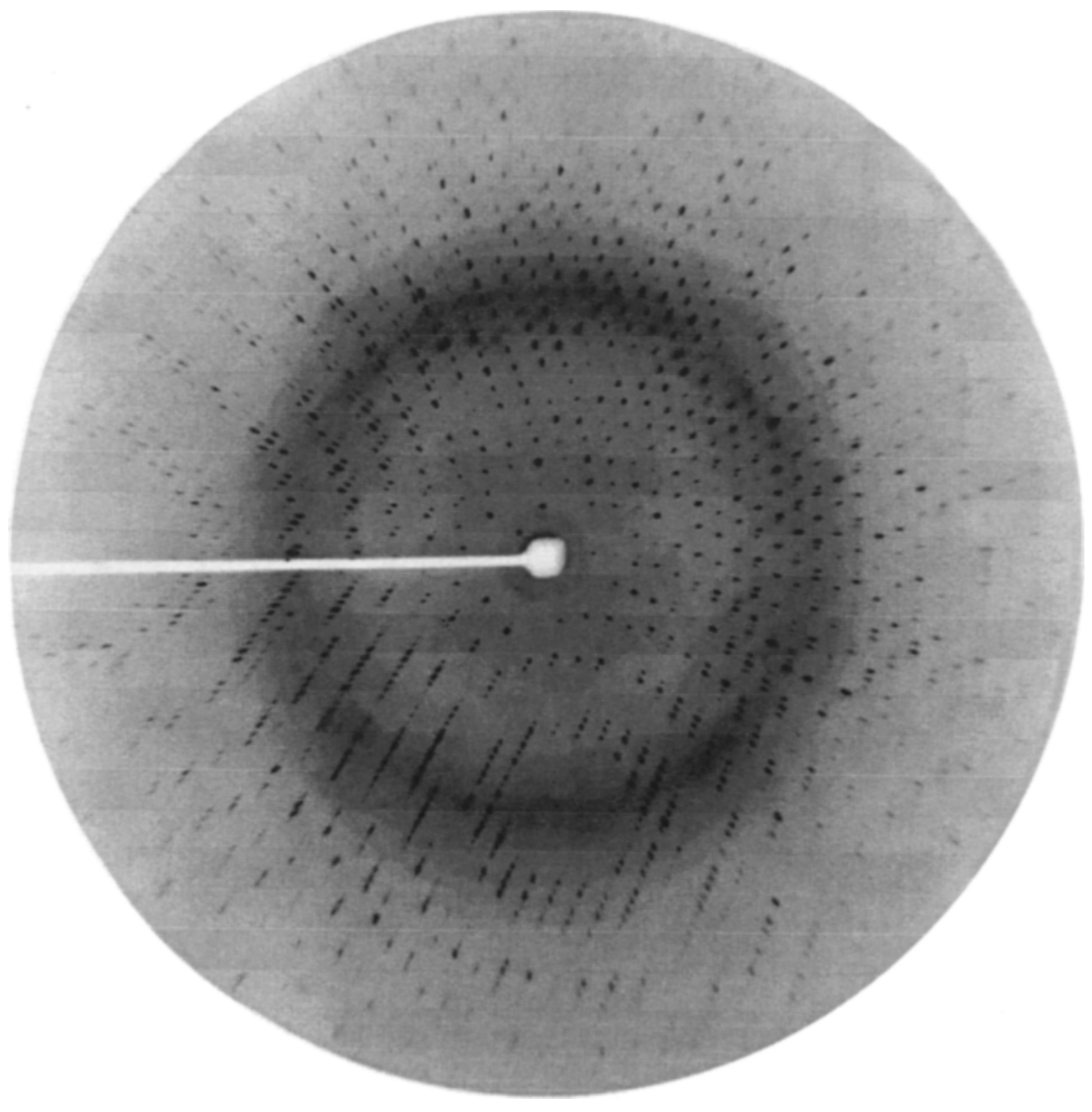

FIGURE 6. Rotation-diffraction pattern from a crystal of TAF42/TAF62, using the multilayer monochromator at a wavelength of $1.4 \AA$. Note the radial streaks of the Bragg spots, which get longer away from the center of the pattern.

One can see, in Fig. 6, that the Bragg spots are indeed radially streaked, more so at the edges of the pattern than closer to the center. In order to make use of this pattern in a structure solution, the Bragg spots must be indexable and their integrated intensities reduceable. The data reduction must take account of the radial elongation of the spots brought about by the anisotropic reciprocal space resolution function, and be able to separate closely spaced or slightly overlapping spots. The crystallography data reduction computer program MOSFLM [22] was used for this purpose. Two data sets were collected, one for which the minimum d-spacing resolution was $3 \AA$ and the other for which it was $2 \AA$. The latter set, being measured to a smaller $\mathrm{d}$-spacing resolution (i.e. to higher $q$ values), was expected to pose more of a data reduction challenge, because of a larger fraction of closely spaced Bragg spots at higher $q$ values. This was indeed confirmed, via comparison of the determined values of $R_{s y m}$ for each data set. $\mathbf{R}_{s y m}$ is a figure of merit that bears upon the uniformity of intensities of symmetry-equivalent Bragg spots; the lower the value of $R_{\text {sym }}$, the better the quality of the data set. The lower resolution (to $3 \AA$ ) data set gave an $R_{\text {sym }}$ of $4.3 \%$, and the higher resolution (to $2 \AA$ ) set gave an $R_{\text {sym }}$ of $9.4 \%$. The lower resolution set is regarded as rather good, whereas the latter might be of questionable value to a structure solution; the $2.4 \AA$ resolution data set collected on the bending magnet beam line where the crystal structure was solved gave an $R_{s y m}$ of $7.6 \%$. It is clear that use of a multilayer monochromator with a slightly narrower wavelength resolution than for the one employed would have resulted in a superior high-resolution data set. Plans are afoot to repeat the experiment with use of the new multilayer pair presently in hand, which has a relative wavelength resolution of $1.1 \times 10^{-2} \mathrm{FWHM}$. 


\section{OUTLOOK}

Broad bandwidth multilayer monochromators can be employed on synchrotron $\mathrm{x}$-ray beam lines to replace narrow bandwidth silicon or germanium crystal monochromators, resulting in substantial intensity gains (as much as 100) at the expense of photon energy and reciprocal space resolution. Experimental applications that do not require high resolution, such as small angle $x$-ray scattering and some crystallography, might profit from the use of such monochromators, for they may make studies of weakly diffracting samples feasible. A bending magnet beam line equipped with a multilayer monochromator may deliver a comparable or higher intensity than an insertion device beam line equipped with a silicon monochromator. This has been demonstrated on the NSLS X20C bending magnet beam line for several years, and multilayer monochromators have recently been installed on bending magnet beam lines X27A and X27C. On insertion device beam lines such as X25, a multilayer monochromator could allow the capability to pursue experiments that could not be pursued with use of a silicon monochromator, even with the enhanced brightness of the insertion device.

\section{ACKNOWLEDGMENTS}

We gratefully acknowledge discussions with and advice from Brian Stephenson, Jim Wood, Jerry Hastings, and Peter Siddons. Technical assistance was provided by Anthony Lenhard, Rick Greene, and Tom Langdon. This work was supported in part by the US Department of Energy under Contract No. DE-AC02-76CH00016. E.D. acknowledges the support of the Natural Sciences and Engineering Research Council of Canada. S.K.B. is an investigator in the Howard Hughes Medical Institute.

\section{REFERENCES}

1. Underwood, J.H. and Barbee, T.W., Appl. Opt. 20, 3027-3034 (1981), and references therein.

2. Bilderback, D.H., Lairson, B.M., Barbee, T.W., Ice, G.E., and Sparks, C.J., Nucl. Instrum. Methods 208, 251-261 (1983).

3. This has been demonstrated with a double multilayer monochromator installed on the microfocus undulator beam line at ESRF, described in Deschamps, P., Engström, P., Fiedler, S., Riekel, C., Wakatsuki, S., Høghøj, P., and Ziegler, E., J. Synchrotron Rad. 2, 124-131 (1995).

4. Freund, A.K., Nucl. Instrum. Methods Phys. Res. A266, 461-466 (1988).

5. Hohlwein, D., Siddons, D.P., and Hastings, J.B., J. Appl. Cryst. 21, 911-915 (1988).

6. X-ray reflectivity calculations for single crystals were made with use of computer software written by the first author. All $\mathrm{x}$-ray reflectivity calculations for multilayers, that are shown in figures in this article, were made with use of computer software operating under the auspices of the Lawrence Berkeley Laboratory Center for X-Ray Optics World Wide Web site, http://www-cxro.lbl.gov.

7. Ziegler, E., Opt. Eng. 34, 445-452 (1995).

8. Stephenson, G.B., Nucl. Instrum. Methods Phys. Res. A266, 447-451 (1988).

9. Berman, L.E., Hastings, J.B., Oversluizen, T., and Woodle, M., Rev. Sci. Instrum. 63, 428-432 (1992).

10. Ziegler, E., Marot, G., Freund, A.K., Joksch, St., Kawata, H., Berman, L.E., and Iarocci, M., Rev. Sci. Instrum. 63, 496-500 (1992).

11. Song, S., Mochrie, S.G.J., and Stephenson, G.B., Phys. Rev. Lett. 74, 5240-5243 (1995).

12. Dierker, S.B., Pindak, R., Fleming, R.M., Robinson, I.K., and Berman, L.E., Phys. Rev. Lett. 75, 449-452 (1995).

13. J.L. Wood, Product Manager, Osmic, Inc., 1788 Northwood Drive, Troy, Michigan 48084, USA.

14. Smither, R.K., Forster, G.A., Bilderback, D.H., Bedzyk, M., Finkelstein, K., Henderson, C., White, J., Berman, L.E., Stefan, P., and Oversluizen, T., Rev. Sci. Instrum. 60, 1486-1492 (1989).

15. Hart, M., Nucl. Instrum. Methods Phys. Res. A297, 306-311 (1990).

16. Berman, L.E., Rev. Sci. Instrum. 66, 2041-2047 (1995).

17. Berman, L.E. and Hastings, J.B., Proc. SPIE 1739, 489-501 (1993).

18. Hart, M., Rodrigues, A.R.D., and Siddons, D.P., Acta Cryst. A40, 502-507 (1984).

19. Berman, L.E., Durbin, S.M., and Batterman, B.W., Nucl. Instrum. Methods Phys. Res. A241, 295-301 (1985).

20. Tsui, O.K.C., Mochrie, S.G.J., and Berman, L.E., J. Synchrotron Rad., to be published (1997).

21. Xie, X., Kokubo, T., Cohen, S.L., Mirza, U.A., Hoffman, A., Chait, B.T., Roeder, R.G., Nakatani, Y., and Burley, S.K., Nature 380, 316-322 (1996).

22. Written by A. Leslie, and for which treatment of the wavelength dispersion is based on formalism described in Greenhough,T.J. and Helliwell, J.R., J. Appl. Cryst. 15, 338-351 (1982); ibid 493-508 (1982); A. Leslie, "Data Collection and Processing," Proceedings of the CCP4 Study Weekend at Daresbury Laboratory, 29-30 January 1993, compiled by L. Sawyer, N. Isaac, S. Bailey, pp. 44-51. 